\title{
O estado da arte da pesquisa acerca da violência doméstica contra a mulher no Brasil
}

\author{
The state of the art of research on domestic violence against women in Brazil \\ El estado del arte de la investigación acerca de la violencia doméstica contra las mujeres en Brasil
}

Recebido: 07/12/2021 | Revisado: 15/12/2021 | Aceito: 17/12/2021 | Publicado: 02/01/2022

Lucilla Vieira Carneiro

ORCID: https://orcid.org/0000-0002-6988-2297 Universidade Federal da Paraíba, Brasil

E-mail: lucilla.vc@hotmail.com

Vitória Polliany de Oliveira Silva

ORCID: https://orcid.org/0000-0003-1363-6940 Universidade Federal da Paraíba, Brasil E-mail: vitoriapolliany1@gmail.com

Luiz Henrique da Silva

ORCID: https://orcid.org/0000-0003-2244-5548 Universidade Federal da Paraíba, Brasil

E-mail: luizenf2014.2@gmail.com

Ana Maria Gondim Valença

ORCID: https://orcid.org/0000-0001-8460-3981 Universidade Federal da Paraíba, Brasil

E-mail: anamvalenca@gmail.com

Juliana Sampaio

ORCID: https://orcid.org/0000-0003-0439-5057 Universidade Federal da Paraíba, Brasil

E-mail: julianasmp@hotmail.com

Hemílio Fernandes Campos Coêlho

ORCID: https://orcid.org/0000-0002-7140-3590 Universidade Federal da Paraíba, Brasil E-mail: hemilio@gmail.com

\begin{abstract}
Resumo
Objetivo: Verificar as evidências científicas reveladas por pesquisas de identificação do perfil da violência doméstica contra a mulher (VDCM) no Brasil. Metodologia: Trata-se de um estudo descritivo do tipo revisão integrativa da literatura. Foi estabelecida a utilização dos descritores: "violência contra a mulher" AND "violência doméstica", aplicados nas seguintes bases de dados: Literatura Latino-Americana e do Caribe em Ciências da Saúde (LILACS), Medical Literature Analysis and Retrieval System Online (MEDLINE) e Scientific Eletronic Library Online (SCIELO). Foram incluídos artigos completos relacionados aoobjeto de pesquisa, nos idiomas de português, inglês e espanhol, indexados nos referidos bancos de dados no período entre 2011 e 2021. Os critérios de exclusão para a amostra foram: artigos de revisão, dissertações, teses e pesquisas realizadas em outros países. Resultados: As buscas nas bases de dados resgataram um total de 415 artigos, dos quais 12 foram incluídos na amostra. Identificou-se a predominância de estudos publicados em 2017. Observou-se a caracterização do agressor, bem como do tipo de violência perpetrada contra a mulher como os principais achados em comum. Conclusão: As evidências científicas demonstraram que o agressor normalmente é do sexo masculino, onde na maioria dos casos trata-se do cônjuge/companheiro da mulher. Quanto ao perfil do tipo de VDCM perpetrada, prevalece a violência psicológica/moral, seguida da violência física e sexual. Somado a isso, o uso de álcool e outras drogas pelos companheiros, demonstrou ser um fator desencadeante para a VDCM. Percebeu-se a deficiência na efetivação de políticas públicas de saúde frente o agravo.
\end{abstract}

Palavras-chave: Violência contra a mulher; Gênero; Políticas públicas de saúde.

\begin{abstract}
Objective: Verifying the scientific evidence revealed by surveys of identify the profile of domestic violence against women (DVAW)in Brazil. Methodology: This is a descriptive study of the integrative review type of the literature. The use of the descriptors: "violence against women" AND "domestic violence", applied in the following databases was established: Latin American and Caribbean Literature onHealth Sciences (LILACS), Medical Literature Analysis and Retrieval System Online (MEDLINE) and Scientific Electronic Library Online (SCIELO). Complete articles related to the research object were included, in Portuguese, English and Spanish languages, indexed in these databases in the period between 2011 and 2021. Exclusion criteria for the sample were: review articles, dissertations, theses and research conducted in other countries. Results: The searches in the databases rescued a total of 415 articles, of which 12 were included in the sample. The predominance of studies published in 2017 was identified. In the studies, it was
\end{abstract}


observed the characterization of the aggressor, as well as the type of violence perpetrated against women as the main findings in common. Conclusion: Scientific evidence has shown that the aggressor is usually male, where in most cases it is the spouse/partner of thewoman. As for the profile of the type of DVAW perpetrated, psychological/moral violence prevails, followed by physical and sexual violence. In addition, the use of alcohol and other drugs by the partners, proved to be a triggering factor for The DVAW. It was noticed deficiency in the implementation of public health policies in the face of the disease.

Keywords: Violence against women; Gender; Public health policies.

\section{Resumen}

Objetivo: Verificar la evidencia científica revelado por encuestas de identificar el perfil de violencia doméstica contra las mujeres (VDCM) en Brasil. Metodología: Se trata de un estudio descriptivo del tipo de revisión integradora de la literatura. Se estableció el uso de los descriptores: "violencia contra la mujer" AND "violencia doméstica",aplicadas en las siguientes bases de datos: Literatura Latinoamericana y del Caribe en Ciencias de la Salud (LILACS), Medical Literature Analysis and Retrieval System Online (MEDLINE) e Scientific Eletronic Library Online (SCIELO). Se incluyeron artículos completos relacionados con el objeto de investigación, en portugués, inglés y español, indexados en estas bases de datos en el periodo comprendido entre 2011 y 2021. Los criterios de exclusión para la muestra fueron: artículos de revisión, disertaciones, tesis e investigaciones realizadas en otros países. Resultados: Las búsquedas en las bases de datos rescataron un total de 415 artículos, de los cuales 12 fueron incluidos en la muestra. Se identificó el predominio de los estudios publicados en 2017. En los estudios, se observó la caracterización del agresor, así como el tipo de violencia perpetrada contra las mujeres como los principales hallazgos en común. Conclusión: La evidencia científica ha demostrado que el agresor suele ser varón, donde en la mayoría de los casos es el cónyuge/pareja de la mujer. En cuanto al perfil del tipo de VDCM perpetrado, prevalece la violencia psicológica/moral, seguida de la violencia físicay sexual. Además, el consumo de alcohol y otras drogas por parte de las parejas, resultó ser un factor desencadenante para el VDCM. Se notó la deficiencia en la implementación de políticas de salud pública frente a la enfermedad.

Palabras clave: Violencia contra la mujer; Género; Políticas de salud pública.

\section{Introdução}

A violência doméstica contra a mulher (VDCM) é um dos principais problemas sociais e de saúde pública no mundo, tornando-se uma das causas crescentes de morbimortalidade do público feminino, perpassando por todas as classes sociais (Jesus et al., 2020).

A VDCM é um tipo de relação social historicamente delimitada, culturalmente legitimada e cultivada, na qual a mulher está exposta a agressões físicas e verbais, tanto no espaço público como privado. As consequências da violência sofrida pela mulher materializam-se em agravos biológicos, psicológicos e sociais, que persistem e se multiplicam ao longo da história, indicando a importância do tema e a necessidade de investigá-lo (Lucena et al., 2016).

Sabe-se que esse tipo de violência se concretiza frequentemente no domínio privado. Nesse ínterim, a pandemia da Covid-19 pode ter aumentado ainda mais a incidência da VDCM, tendo em vista que a necessidade do isolamento social intensificou o convívio com a pessoa agressora, elevando o risco de uma mulher sofrer algum tipo de agressão dentro da própria casa. Além disso, ressalta-se que a crise sanitária, econômica e social vivenciada nos últimos anos no Brasil, bem como, suas necessárias medidas de enfrentamento podem aumentar o risco de violência contra a mulher devido a sua menor visibilidade. (Reigada \& Smiderle, 2021).

No Brasil, em agosto de 2006, foi sancionada a Lei 11.340, denominada Lei Maria da Penha, com o intuito de incrementar e destacar o rigor das punições para esse tipo de crime. Mais recente ainda, em março de 2015, foi sancionada a Lei 13.104/2015, denominada Lei do Feminicídio, classificando-o como crime hediondo e com agravantes quando acontece em situações específicas de vulnerabilidade (gravidez, menor de idade e na presença de filhos) (Lucena \& Deininger, 2018).

De acordo com a Lei Maria da Penha (Lei $\mathrm{n}^{\circ}$ 11.340/2006), a violência doméstica e familiar contra a mulher é “qualquer ação ou omissão baseada no gênero que lhe cause morte, lesão, sofrimento físico, sexual ou psicológico e dano moral ou patrimonial" (Brasil, 2010). A violência pode ser perpetrada por familiares, companheiros ou ex-companheiros que vivam ou não no mesmo ambiente que a mulher, podendo ser cometida dentro ou fora deste (Batista et al., 2018). 
No que concerne às políticas públicas, destaca-se a implementação da Política Nacional de Enfrentamento à Violência contra as Mulheres, que consiste num acordo entre as três esferas do governo para planejamento de ações, e estabelecimento de princípios e diretrizes para prevenção e combate à violência e para a garantia de direitos às mulheres. Assim, a Rede de Enfrentamento à Violência Contra a Mulher visa desenvolver estratégias efetivas para o empoderamento feminino, garantia de seus direitos, responsabilização dos agressores e promoção da assistência qualificada às mulheres em situação de violência (Brasil, 2011).

Segundo Lima et al. (2021), cerca de três ou mais brasileiras acima de 16 anos de idade estão expostas à agressão. Dessa totalidade, cerca de 503 milhões de mulheres são vítimas de agressões físicas e 40\% são mulheres acima dos 16 anos de idade que já sofreram algum tipo de assédio.

De acordo com dados do Atlas da Violência, divulgado em 2019, 4.936 mulheres foram assassinadas no Brasil em 2017, cerca de 13 mulheres por dia, o maior número desde 2007. Esse fato alarmante corresponde a uma taxa de 4,7 mortes para cada 100 mil mulheres. Na década (2007-2017) houve um crescimento expressivo de 30,7\% no número de homicídios de mulheres no país, assim como no último ano da série, 2017, que registrou aumento de 6,3\% em relação ao anterior (Brasil, 2019).

Por sua complexidade, a VDCM ainda representa um desafio para o setor saúde. Entre as principais dificuldades para ultrapassar tal desafio encontram-se, os obstáculos para a sua identificação, tais como os fatores de ordem cultural, político, econômico e sexual, além da falta de orientação tanto das usuárias quanto dos profissionais de saúde (Batista et al., 2018).

Diante do exposto, o desenvolvimento do estudo justifica-se pela importância de caracterizar a produção científica acerca da VDCM no contexto brasileiro, vislumbrando identificar quais fatores estão relacionados a sua ocorrência, assim como aprofundar discussões sobre o impacto desse fenômeno. Assim, tem-se como objetivo verificar as evidências científicas reveladas por pesquisas de identificação do perfil da violência doméstica.

\section{Metodologia}

Trata-se de um estudo descritivo do tipo revisão integrativa da literatura, cujo método possibilita sintetizar pesquisas já concluídas e adquirir resultados através de um tema de interesse (Pereira et al., 2018). Assim, emerge a seguinte questão norteadora que fundamenta o mérito desta investigação: Qual a tendência dos estudos primários de investigação acerca do perfil da VDCM no Brasil, no período compreendido entre 2011 e 2021 ?

A coleta de dados ocorreu no mês de setembro de 2021. Realizou-se a busca dos artigos por meio da Biblioteca Virtual de Saúde (BVS) e Scientific Eletronic Library Online (SCIELO), sendo selecionadas na BVS as seguintes bases de dados: Literatura Latino-Americana e do Caribe em Ciências da Saúde (LILACS) e Medical Literature Analysis and Retrieval System Online (MEDLINE). Foram utilizados os seguintes descritores: "violência contra a mulher" e "violência doméstica", associados ao operador booleano AND.

Foram incluídos artigos completos relacionados à questão norteadora de pesquisa, nos idiomas de português, inglês e espanhol, indexados nos referidos bancos de dados no período citado. Os critérios de exclusão para a amostra foram: artigos de revisão, dissertações, teses e pesquisas realizadas em outros países.

O processo de elaboração do estudo foi composto por seis etapas: elaboração da questão norteadora da pesquisa; busca dos artigos nas bases de dados indexadas; leitura e avaliação dos artigos selecionados; discussão dos resultados; síntese do conhecimento e apresentação da revisão integrativa (Koller et al., 2014).

Para sistematizar o processo de seleção dos artigos, utilizou-se a metodologia Preferred Reporting Items for Systematic Reviews and Meta-Analyses (PRISMA) (Liberati et al., 2009). As etapas deste processo estão descritas em um fluxograma (Figura 1). 
Figura 1: Fluxograma do processo de seleção dos artigos baseado no método PRISMA.
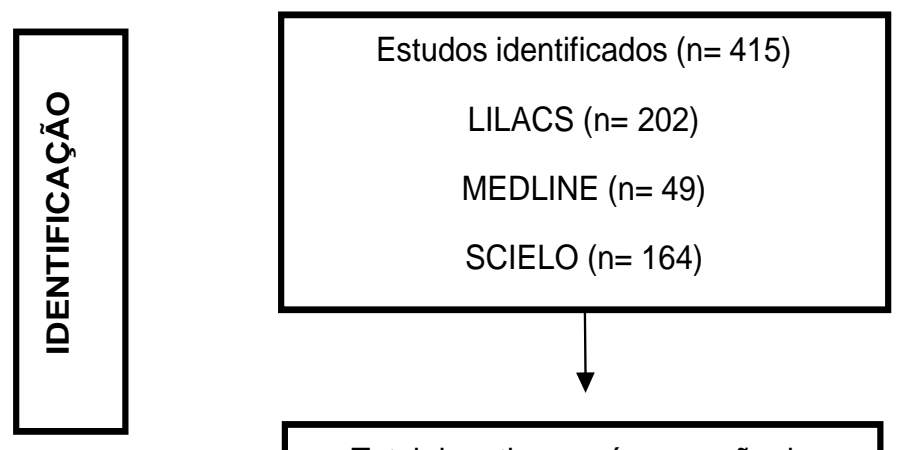

Total de artigos após remoção de duplicatas $(n=407)$
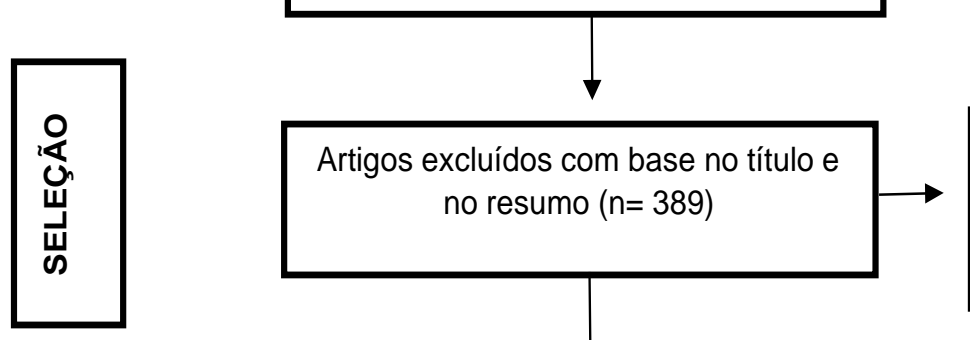

Motivos: Temas que não atingiram os objetivos do estudo, dissertações, teses, estudos de revisão e pesquisas realizadas em outros países.
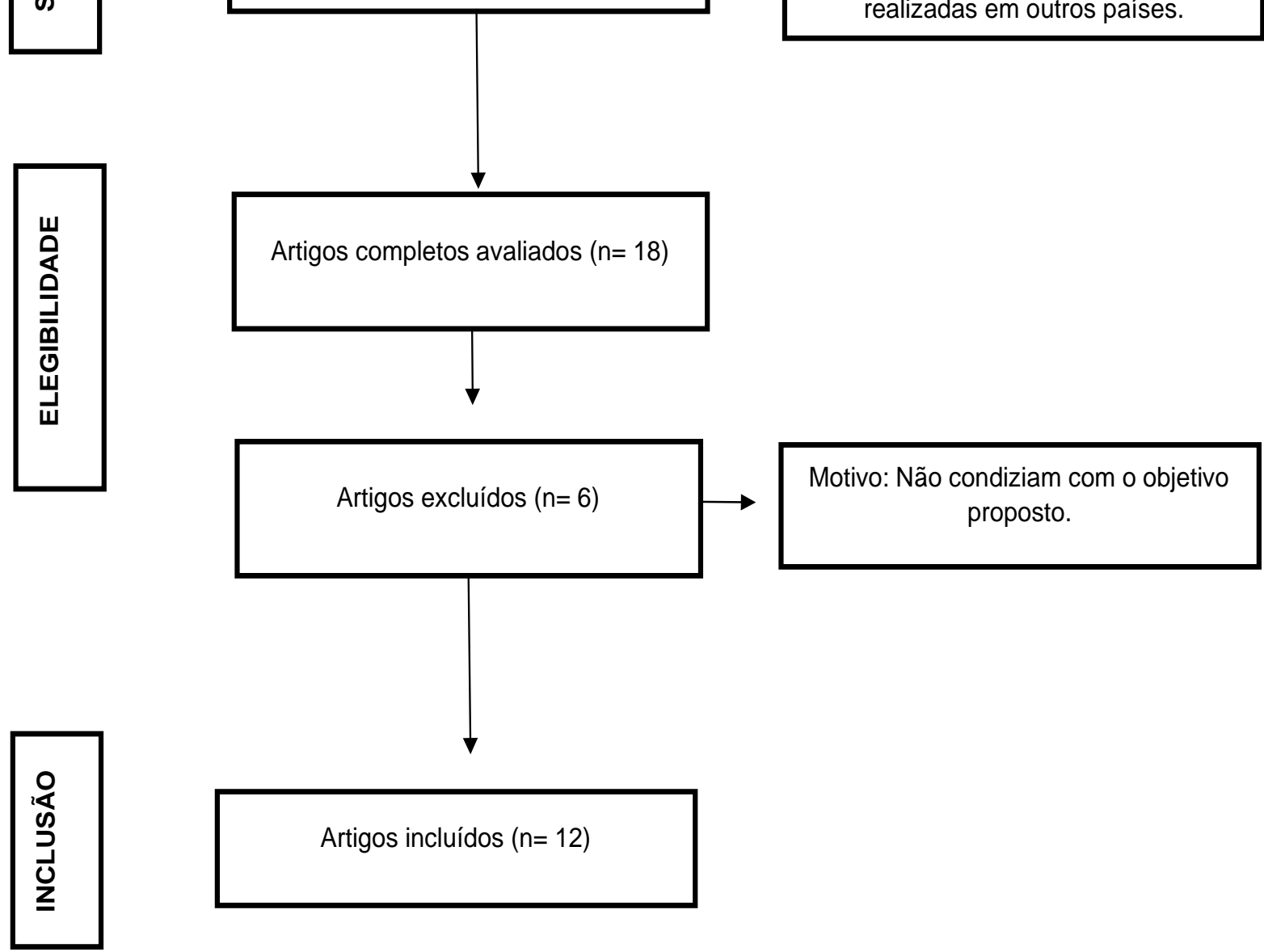

Fonte: Dados da Pesquisa, 2021.

\section{Resultados e Discussão}

As buscas nas bases de dados resgataram um total de 415 artigos, dos quais 12 foram incluídos na amostra. O Quadro 1 mostra os metadados dos artigos selecionados: autor, ano de publicação, delineamento do estudo, amostra, instrumento de 
coleta de dados e principais resultados. Os estudos que compuseram a amostra, foram distribuídos conforme o ano de publicação, numa escala crescente do mais antigo para o mais recente.

Quadro 1. Características gerais dos estudos selecionados para a amostra.

\begin{tabular}{|c|c|c|c|}
\hline $\mathbf{N}^{\mathbf{0}}$ & Autor/ Ano & $\begin{array}{c}\text { Delineamento, Amostra, } \\
\text { Instrumento }\end{array}$ & Principais resultados \\
\hline 1 & Vale et al., 2013 & $\begin{array}{l}\text { Quantitativo } \\
\text { Descritivo } \\
\mathrm{n}=211 \\
\text { Entrevista }\end{array}$ & $\begin{array}{l}\text { A maioria das mulheres do estudo em situação de } \\
\text { violência eram casadas, na faixa etária adulta e não } \\
\text { trabalhavam. }\end{array}$ \\
\hline 2 & Zancan et al., 2013 & $\begin{array}{l}\text { Qualitativo } \\
\text { Descritivo } n=4 \\
\text { Entrevista }\end{array}$ & $\begin{array}{l}\text { Foram identificadas ocorrências de violênciafísica, } \\
\text { psicológica e sexual. }\end{array}$ \\
\hline 3 & Gomes et al., 2014 & $\begin{array}{l}\text { Qualitativo } \\
\text { Descritivo } \\
\mathrm{n}=6 \\
\text { Entrevista }\end{array}$ & $\begin{array}{l}\text { As agressões foram evidenciadas por empurrões, tapas, } \\
\text { murros, arma branca e armade fogo. }\end{array}$ \\
\hline 4 & Vigário \& Paulino-Pereira, 2014 & $\begin{array}{l}\text { Qualitativo } \\
\text { Pesquisa-ação } \\
\mathrm{n}=3 \\
\text { Diário de Campo e Escuta } \\
\text { Terapêutico-educativa }\end{array}$ & $\begin{array}{l}\text { As mulheres apontaram o álcool como estopim da } \\
\text { violência física e psicológica que viviam noseu cotidiano. }\end{array}$ \\
\hline 5 & Oliveira et al., 2015 & $\begin{array}{l}\text { Qualitativo } \\
\text { Pesquisa fenomenológican }=10 \\
\text { Entrevista }\end{array}$ & $\begin{array}{l}\text { O agressor era predominantemente do sexo masculino, na } \\
\text { maioria dos casos tratava-se do cônjuge e destacava-se } \\
\text { a violência de } \\
\text { repetição. }\end{array}$ \\
\hline 6 & Silva et al., 2015 & $\begin{array}{l}\text { Qualitativo Exploratório } \\
\text { descritivon=406 } \\
\text { Entrevista }\end{array}$ & $\begin{array}{l}\text { Com relação aos tipos de violência, } 67 \% \text { relataram ter } \\
\text { sofrido violência do tipo psicológica e } 33 \% \text { do tipo física } \\
\text { e psicológica. }\end{array}$ \\
\hline 7 & Ferreira et al., 2016 & $\begin{array}{l}\text { Quanti-qualitativo } \\
\text { Exploratório descritivo } \\
\mathrm{n}=197 \\
\text { Dados de prontuários }\end{array}$ & $\begin{array}{l}\text { Mais de } 2 / 3 \text { da amostra }(86,3 \%) \text { foi agredidapor } \\
\text { parceiro fixo (marido ou namorado). }\end{array}$ \\
\hline 8 & Garcia et al., 2016 & $\begin{array}{l}\text { Quantitativo Caso } \\
\text { e controle } \\
\mathrm{n}=623 \text { casos e } 10.120 \text { controlesDados } \\
\text { do Inquérito VIVA }\end{array}$ & $\begin{array}{l}\text { A violência doméstica e familiar teve o consumo de } \\
\text { bebida alcoólica como fator fortemente associado. }\end{array}$ \\
\hline 9 & Bozzo et al., 2017 & $\begin{array}{l}\text { Quantitativo } \\
\text { Documental } \\
\mathrm{n}=5643 \\
\text { Dados do SINAN }\end{array}$ & $\begin{array}{l}\text { Constatou-se que a violência psicológica/moral se } \\
\text { destacou com } 4640(82,33 \%) \text { casos, seguida da violência } \\
\text { física, com } 2792(49,48 \%) \text { eventos. }\end{array}$ \\
\hline 10 & Echeverria et al., 2017 & $\begin{array}{l}\text { Qualitativo } \\
\text { Descritivo } \mathrm{n}=5 \\
\text { Entrevista }\end{array}$ & $\begin{array}{l}\text { Predominou-se a violência psicológica, com cinco } \\
\text { relatos, a física, com três relatos, um relato de violência } \\
\text { moral e um de violência } \\
\text { patrimonial. }\end{array}$ \\
\hline 11 & Lucena et al., 2017 & $\begin{array}{l}\text { Quantitativo } \\
\text { Inquérito domiciliar de base } \\
\text { populacional, transversal } n=424 \\
\text { Questionários }\end{array}$ & $\begin{array}{l}\text { Verificou-se que mulheres com avaliação da satisfação da } \\
\text { QV aumentada apresentaram chance } 6,0 \% \text { menor de } \\
\text { sofrer violência doméstica. }\end{array}$ \\
\hline 12 & Fiorotti et al., 2018 & $\begin{array}{l}\text { Quantitativo Epidemiológico, } \\
\text { observacional, transversaln }=302 \\
\text { Entrevista }\end{array}$ & $\begin{array}{l}\text { Observou-se que mulheres com idade entre } 31 \text { a } 43 \text { anos e } \\
\text { que tiveram três ou mais gestações possuem } 50,0 \% \text { e } \\
80,0 \% \text {, respectivamente, mais prevalência de relato de } \\
\text { maus-tratos ao longo da vida. }\end{array}$ \\
\hline
\end{tabular}

Fonte: Dados da Pesquisa (2021).

No que concerne ao ano de publicação, identificou-se a predominância de estudos publicados em 2017 (n=3). Ressalta- se que foi observada ausência de publicações nacionais nos últimos três anos que respondem à questão norteadora do estudo, nas bases de dados pesquisadas. Em relação à técnica de coleta de dados, houve o predomínio de entrevistas (n=7).

Os estudos apresentam a caracterização do agressor, bem como, do tipo de violência perpetrada contra a mulher como os principais achados em comum. Em relação à tipologia da violência perpetrada contra a mulher, quatro estudos apresentaram a violência psicológica como a mais prevalente. Quanto ao perfil do agressor, constatou-se que normalmente trata-se do cônjuge da mulher. Somado a isso, observou-se que o predomínio da faixa etária da mulher em situação de violência varia entre 30 e 45 anos. 
Desse modo, a partir das leituras dos respectivos artigos identificaram-se quatro categorias temáticas: Tipologia da violência perpetrada, Perfil das mulheres em situação de violência doméstica, Perfil do agressor e fatores desencadeantes para a VDCM e Redes de apoio.

\section{Categoria 1 - Tipologia da violência perpetrada}

A VDCM constitui um grave problema de saúde pública, capaz de propiciar severas repercussões biopsicossociais na vida das vítimas do fenômeno e entre o seu núcleo familiar. Vale enfatizar, que muitas vezes as mulheres desconhecem os tipos de violência que podem sofrer, ressaltando comumente apenas a violência física e psicológica. Assim, destaca-se que os agravos provocados a partir da violência doméstica vão além dos sintomas físicos, perpassando por transtornos e consequências psicológicas, além da diminuição da qualidade de vida (Silva et al., 2015).

Percebe-se que normalmente as agressões ocorrem com maior frequência nas residências e as partes do corpo das mulheres mais atingidas são a cabeça e os membros superiores. Quanto aos horários das agressões, a maior parte ocorre durante a noite ou madrugada (Garcia et al., 2016). Comumente, a força física mostra-se ser o meio mais utilizado para promover a VDCM (Ferreira et al., 2016).

Segundo Vale et al. (2013), dentre as repercussões psicoemocionais da violência doméstica sobre as mulheres, destacam-se os sentimentos de tristeza, raiva e depressão. Nesse sentido, de acordo com Jesus et al. (2020), a violência psicológica pode acarretar danos significativos, apresentando os transtornos mentais como principais implicações.

Além do mais, a ocorrência de impactos sociais é bastante comum, já que esse fenômeno interfere em grande proporção na produtividade e na rotina laboral das mulheres. Nesse contexto, mediante aos elevados índices desse agravo e devido aos seus impactos, torna-se crucial a atuação eficiente da equipe multidisciplinar de saúde no enfrentamento dessa situação, assim como do desenvolvimento de políticas públicas capazes de modificar esse cenário (Echeverria et al., 2017).

Vale ressaltar, que as violências psicológica, física e sexual apresentam associação significativa com a religião evangélica. Nessa perspectiva, a busca pela religião se apresenta na literatura como uma forma de suportar ou superar a violência vivida (Santos et al., 2020).

Nessa perspectiva, Oliveira et al. (2015) destacam que as pesquisas realizadas junto a mulheres em situação de violência permitem desvelar esse fenômeno em suas múltiplas formas de expressão. Outrossim, a análise do fenômeno violência doméstica emergem vivências que permitem diagnosticar o medo experienciado por mulheres, a convivência com as lesões físicas e danos psicológicos e o difícil processo até a decisão de denunciar após a violência vivida.

Destarte, percebe-se que a VDCM é um amplo problema enraizado na nossa cultura patriarcal, resultante das desigualdades nas relações de gênero, ocorrendo quase sempre em função de princípios históricos e da construção social que privilegia o homem. De acordo com Lucena et al. (2016), o patriarcado é um sistema de dominação e opressão, o qual se constitui como uma forma de submissão das mulheres em relação aos homens, fazendo reverberar as iniquidades de poder expressas nas relações desiguais entre homens e mulheres. Por isso, ressalta-se a importância de pontuarmos aspectos primordiais dessa temática, como a invisibilidade da VDCM num contexto cultural machista, a invisibilidade do privado, a visibilidade conquistada através da luta do movimento feminista e a necessidade da atuação de órgãos/serviços públicos que possam intervir nesses casos (Jesus et al., 2020).

\section{Categoria 2 - Perfil das mulheres em situação de violência doméstica}

Comumente, o perfil da mulher vítima de violência doméstica está fortemente associado a piores condições socioeconômicas. Desse modo, a ocorrência do fenômeno é mais frequente entre aquelas com menor escolaridade e negras 
(D’Oliveira et al., 2009). Contudo, acredita-se que mulheres com níveis educacionais mais altos possuem maior habilidade para reconhecer e romper relacionamentos abusivos (Santos et al., 2020).

Além disso, a maioria das mulheres em situação de violência doméstica não trabalham. Vale ressaltar, que o fato de a mulher não trabalhar ou não possuir qualquer renda pode acentuar ainda mais o agravo (Vale et al., 2013). Segundo Oliveira et al. (2015), todo o contexto da VDCM gera medo, dificuldade de rompimento com a pessoa causadora da violência, insegurança financeira, além do comportamento de submissão para com o agressor.

Quanto ao estado civil, pesquisa realizada por Lucena et al. (2017) em João Pessoa - PB, apontou que 56\% das mulheres em situação de violência doméstica viviam com o companheiro. Em contraponto, difere do estudo de Leite et al. (2017), cujos resultados apontaram maior prevalência desse agravo entre mulheres que se declararam divorciadas ou separadas. Esses dados demonstram, que muitas mulheres conseguem se libertar da situação de violência doméstica pelo parceiro íntimo, rompendo o estereótipo de que o fenômeno é uma situação irremediavelmente crônica (Santos et al., 2020).

Outro fato importante, é que mulheres que tiveram três ou mais gestações, possuem 50,0\% e 80,0\%, respectivamente, mais prevalência de relato de maus-tratos ao longo da vida. Além disso, entre as puérperas a violência doméstica também é significante. Todavia, a prevalência dessa violência é 4,6 vezes mais comum entre puérperas sem companheiros, quando comparado àquelas mulheres que possuem companheiro noperíodo gestacional. Sendo assim, nota-se que mulheres gestantes em situação conjugal fragilizada são mais vulneráveis a discriminações e preconceitos arraigados em nossa cultura, que podem resultar na ocorrência de violência. Contudo, a inserção da temática na prática assistencial é fundamental, para a adequada promoção de cuidado às vítimas (Fiorotti et al., 2018).

Ademais, quanto mais a mulher necessita de atendimento médico para tratamento da saúde, maior é a chance de ocorrência de violência. Dessa maneira, percebe-se que mulheres poliqueixosas nos serviços de saúde podem ser um alerta de violência doméstica. Somado a isso, observou-se que quanto maior é a satisfação da qualidade de vida entre as mulheres, menor é a probabilidade desse tipo de violência (Lucena et al., 2017)

Sabe-se também, que mulheres sob efeitos de álcool se tornam mais vulneráveis à episódios de violência doméstica. Todavia, a relação entre consumo em excesso de álcool e violência doméstica é vastamente conhecida e documentada (Garcia et al., 2016). Na pesquisa de Vieira et al. (2011), identificou-se risco duas vezes e meia maior de sofrer violência doméstica pelo parceiro para mulheres que realizaram uso de álcool. Portanto, a natureza desse fenômeno é multicausal e sofre interferência de diversos fatores. Nesse contexto, vale refletir também que mulheres expostas a situação de violência doméstica estão mais propensas a consumir álcool e drogas ilícitas, como forma de lidar com o evento violento (Santos et al., 2020).

\section{Categoria 3 - Perfil do agressor e fatores desencadeantes para a VDCM}

A maioria das mulheres que sofrem violência doméstica possuem relacionamento fixo com o agressor (marido ou namorado). Entretanto, embora seja em menor proporção, os agressores também podem ser o ex-parceiro, patrão e parentes da vítima (Ferreira et al., 2016).

Em consonância com esses achados, uma investigação com quatro mulheres no interior do Rio Grande do Sul, também apontou o cônjuge como o principal responsável pelas agressões, principalmente quando se encontram sob efeitos de álcool e/ou outras drogas (Zancan et al., 2013).

Um estudo realizado por Brasileiro e Melo (2016), apresentou o perfil dos agressores, onde foi registrado que 55,8\% destes estudaram até o ensino fundamental. Em relação à religião, a maioria dos agressores se denominou cristão, e a idade média deles estava entre 22 e 32 anos. Quanto às profissões dos agressores, destacam-se ocupações específicas, como pedreiro, comerciante, operador de máquinas e serviços gerais. Além disso, destaca-se que o consumo de bebida alcoólica potencializa a discussão, a desavença entre os parceiros, assim como o ciúme. 
Embora o uso de álcool seja apontado na literatura como o principal estopim para a violência doméstica, é mister destacar que o uso dessa substância e/ou outras drogas não produz a violência, mas pode acentuar uma condição que a provoca e com isso constitui apenas um fator contribuinte em uma rede causal complexa, para a determinação da VDCM (Vigário \& Paulino-Pereira, 2014; Garcia et al., 2016).

Dentre os fatores desencadeantes das agressões, o ciúme apresenta destaque, demonstrando a existência preponderante do patriarcado, que se deve ao sentimento de posse do homem sobre a mulher (Ferreira et al., 2016). No mesmo sentido, o ciúme é interpretado como demonstração de carinho e afeto, dificultando o reconhecimento precoce da violência e contribuindo para a naturalização do fenômeno (Haack \& Falcke, 2020).

Desse modo, a literatura científica aponta que a dependência emocional e afetiva pelo cônjuge contribui para que as mulheres continuem no ciclo de violência (Vigário \& Paulino-Pereira, 2014). Somado a isso, as ameaças por parte dos companheiros também propiciam a permanência das mulheres no relacionamento abusivo, podendo favorecer consequentemente a recidiva da violência (Zancan et al., 2013).

Portanto, compreender o perfil do agressor e dos fatores associados à violência doméstica contribuirá para a elaboração e o desenvolvimento de estratégias de prevenção, bem como, de políticas públicas e medidas interventivas para a promoção da saúde dos envolvidos (Vasconcelos et al., 2016).

\section{Categoria 4 - Redes de Apoio}

Apesar do avanço trazido pela Lei Maria da Penha, algumas mulheres não se sentem seguras para a denúncia. Além disso, ainda existem questões estruturais locais que não propiciam esta segurança à mulher em situação de violência e não permitem a aplicação da lei com o devido rigor, a exemplo da ausência de casas de apoio ou abrigo para a mulher manter-se distante do agressor enquanto o caso aguarda uma resolução no âmbito judiciário, além da morosidade na tramitação dos processos (Gomes et al., 2014).

Através da promulgação desta Lei, as Delegacias de Defesa da Mulher (DDM) se tornaram a principal porta de entrada para o acolhimento e atendimento das mulheres vítimas de violência doméstica, porém questiona-se o fato de ficarem abertas apenas durante a semana e no período diurno, de modo a limitar a garantia dos direitos das mulheres. Com isso, muitas vítimas são atendidas em Delegacias que não são preparadas para esse tipo de demanda, dificultando então a incorporação da mulher na rede de atendimento (Machado et al., 2020).

Percebe-se que o apoio social frente a VDCM é fundamental. Entretanto, a maioria das mulheres nessa condição utilizam técnicas individuais de enfrentamento e só decidem buscar ajuda de familiares, amigos, igrejas ou serviços de apoio, quando surgem ameaças de morte por parte do agressor (Souza \& Silva, 2019).

Contudo, existe uma carência ampla de profissionais de saúde habilitados para o estabelecimento de diagnóstico precoce da VDCM. Outro fato agravante, é que o preenchimento da ficha de notificação compulsória de casos ainda não é uma prática cotidiana nos serviços de saúde. Essa realidade demonstra, a necessidade da capacitação desses profissionais frente a temática, bem como do desenvolvimento de políticas públicas de saúde que garantam a assistência contínua às mulheres vítimas de violência doméstica, de modo a efetivar o direito de cidadania (Bozo et al., 2017).

Ressalta-se, inclusive, que na maioria das vezes a própria mulher não é capaz de perceber que está vivendo uma situação de violência e justamente nessas situações é onde a vítima mais necessita de apoio profissional (Machado et al., 2020). Portanto, a atuação dos profissionais de saúde na identificação dos episódios de violência doméstica é fundamental, principalmente na Atenção Básica, já que esse serviço tem como base o seio familiar e o contexto psicossocial que os usuários estão inseridos (Vale et al., 2013). 
Dessa maneira, destaca-se a necessidade de ofertar uma assistência qualificada às mulheres que vivem na situação de violência doméstica, através de uma rede de atendimento que inclui várias portas de entrada: serviços de urgência e emergência de saúde, serviços de assistência social e as DDM, além de recursos comunitários. Somado a isso, é crucial que haja a articulação entre os serviços de urgência e emergência e da atenção primária, possibilitando uma ação efetiva para a integralidade dessas mulheres (Machado et al., 2020).

\section{Conclusão}

As evidências científicas demonstraram que o agressor normalmente é do sexo masculino, onde na maioria dos casos trata-se do cônjuge/companheiro da mulher. Ademais, foi possível identificar que quanto ao perfil do tipo de VDCM perpetrada, prevalece a violência psicológica/moral, seguida da violência física e sexual. Somado a isso, o uso de álcool e outras drogas pelos companheiros, demonstrou ser um fator desencadeante para a violência doméstica. Percebeu-se também, a deficiência na efetivação de políticas públicas de saúde frente o agravo.

Através deste trabalho foi possível mapear a produção científica acerca das pesquisas de diagnóstico sobre VDCM no Brasil, no período pesquisado, onde evidenciou-se o aumento no número de casos desse agravo. Contudo, o referido estudo trouxe diversas contribuições, demonstrando que para enfrentar a VDCM não bastam políticas repressoras, embora sejam importantes, não são suficientes. É preciso desconstruir essa sociedade patriarcal, com políticas de educação, cultura, entre outras, que pautem o tema da desigualdade de gênero e do patriarcado. Assim, com esses achados espera-se cooperar para a ampliação do debate acerca da temática nos diversos âmbitos, tendo em vista que o campo da intervenção precisa atuar de forma preventiva nas medidas de enfrentamento do fenômeno.

Além disso, durante a realização do estudo, destaca-se como limitação a incipiência de pesquisas de campo recentes que contemplem a caracterização do perfil das mulheres em situação de violência doméstica. Portanto, é imprescindível fomentar ações que visem extinguir a exposição das mulheres às situações de violência, considerando as diversidades econômicas, culturais, sociais, étnicas/raciais e de orientação sexual. Assim, destaca-se a importância de promover novos estudos que possam direcionar mecanismos de enfrentamento à VDCM, na perspectiva de identificar os fatores relacionados e direcionar ações que vislumbrem a equidade de gênero, assim como como a garantia da assistência à saúde das mulheres em situação de violência.

\section{Referências}

Batista, K. B. C., Schraiber, L. B., \& D’Oliveira, A. F. P. L. (2018). Gestores de saúde e o enfrentamento da violência de gênero contra as mulheres: as políticas públicas e sua implementação em São Paulo, Brasil. Cadernos de Saúde Pública, 34, e00140017.

Bozzo, A. C. B., Matos, G. C., Beraldi, L. P., \& Souza, M. D. (2017). Violência doméstica contra a mulher: caracterização dos casos notificados em um município do interior paulista. Revista Enfermagem UERJ, 25, e11173.

Brasil (2010). Câmara dos deputados. Lei Maria da Penha nº11.340. Brasília. http://adcon.rn.gov.br/ACERVO/spmrn/DOC/DOC000000000076385.PDF.

Brasil (2019). Ministério do Planejamento Desenvolvimento e Gestão. Atlas da Violência 2019 Ipea $e$ FBSP. http://www.ipea.gov.br/portal/index.php?option=com_content\&view=article\&id=34784\&Ite mid=432.

Brasil (2011). Secretaria de Políticas Para as Mulheres. Presidência da República. Política Nacional de Enfrentamento à Violência Contra as Mulheres/Presidência da República. Brasília. http://www.spm.gov.br/sobre/publicacoes/publicacoes/2011/pactonacional.

Brasileiro, A. E., \& Melo, M. B. (2016). Agressores na violência doméstica: um estudo do perfil sociojurídico. Revista de Gênero, Sexualidade e Direito. Campina Grande, v. 2, n. 2, p. 189-208.

D'Oliveira, A. F. P. L., Schraiber, L. B., França-Junior, I., Ludermir A. B, Portella, A.P, Diniz, C. S., \& Valença, O. (2009). Factors associated with intimate partner violence against Brazilian women. Rev Saúde Pública, 43(2),299-311.

Echeverria, J. G. M.., Oliveira, M. H. B. D., \& Erthal, R. M. D. C. (2017). Violência doméstica e trabalho: percepções de mulheres assistidas em um Centro de Atendimento à Mulher. Saúde em Debate, 41, 13-24.

Ferreira, R. M.., Vasconcelos, T. B. D., Moreira Filho, R. E., \& Macena, R. H. M. (2016). Características de saúde de mulheres em situação de violência doméstica abrigadas em uma unidade de proteção estadual. Ciência \& Saúde Coletiva, 21(12), 3937-3946. 
Fiorotti, K. F., Amorim, M. H. C., Lima, E. D. F. A., Primo, C. C., Moura, M. A. V., \& Leite, F. M. C. (2018). Prevalência e fatores associados à violência doméstica: Estudo em uma maternidade de alto risco. Texto \& Contexto-Enfermagem, 27(3), e0810017.

Garcia, L. P., Duarte, E. C., Freitas, L. R. S. D., \& Silva, G. D. M. D. (2016). Violência doméstica e familiar contra a mulher: estudo de casos e controles com vítimas atendidas em serviços de urgência e emergência. Cadernos de Saúde Pública, 32(4), e00011415.

Gomes, I. C. R., Rodrigues, V. P., Nery, I. G., Vilela, A. B. A., de Oliveira, J. F., \& Diniz, N. M. F. (2014). Enfrentamento de mulheres em situação de violência doméstica após agressão. Revista Baiana de Enfermagem, 28(2), 134-144.

Haack, K. R., \& Falcke, D. (2020). Seria o Ciúme Mediador entre as Experiências na Família de Origem e a Violência Física na Conjugalidade? Psico$U S F, 25(3), 425-437$.

Jesus, L.B., Bizarria, F. P. A., \& Façanha, C. M. H. L. (2020). Violência contra mulher: aspectos normativos e práticas preventivas. Research, Society and Development, v. 9, n. 10, e9249108985.

Koller, S. H.., Couto, M. C. P. P., \& Von Hohendorff, J. (2014). Manual de produção científica. Penso Editora.

Leite, F. M. C., Amorim, M. H. C., Wehrmeister, F. C., \& Gigante, D. P. (2017). Violência contra a mulher em Vitória, Espírito Santo, Brasil. Revista de Saúde Pública, 51, 33 .

Liberati, A., Altman, D. G., Tetzlaff, J., Mulrow, C., Gøtzsche, P. C., Ioannidis, J. P., Clarke, M., Devereaux, P.J., Kleijnen, J., \& Moher, D. (2009). The PRISMA statement for reporting systematic reviews and meta-analyses of studies that evaluate health care interventions: explanation and elaboration. Journal of clinical epidemiology, 62(10), e1-e34.

Lima, S. R., Barros, B., Lagrca, A., Pimentel, A., Martins, J., \& Bueno, S. (2021). Visível e invisível: a vitimização de mulheres no Brasil. (3a ed.). https://forumseguranca.org.br/wp-content/uploads/2021/06/relatorio-visivel-e-invisivel-3ed-2021-v3.pdf

Lucena, K. D. T. D., Deininger, L. D. S. C., Coelho, H. F. C., Monteiro, A. C. C., Vianna, R. P. D. T., \& Nascimento, J. A. D (2016). Análise do ciclo da violência doméstica contra a mulher. J. Hum. Growth Dev, 26 (2), 139-146.

Lucena, K. D. T. D., Vianna, R. P. D. T., Nascimento, J. A. D., Campos, H. F. C., \& Oliveira, E. C. T. (2017). Associação entre a violência doméstica e a qualidade de vida das mulheres1. Revista Latino-Americana de Enfermagem, 25, e2901.

Lucena, K. D.T., \& Deininger, L. S. C. (2018). Ensaios sobre gênero (1ed). Escola de Enfermagem Nova Esperança. https://issuu.com/fechine/docs/ebook_ensaios_sobre_g_nero

Machado, D. F., Almeida, M. A. S. D., Dias, A., Bernardes, J. M., \& Castanheira, E. R. L. (2020). Violência contra a mulher: o que acontece quando a Delegacia de Defesa da Mulher está fechada? Ciência \& Saúde Coletiva, 25(2), 483-494.

Oliveira, P. P. D., Viegas, S. M. D. F., Santos, W. J. D., Silveira, E. A. A. D., \& Elias, S. C. (2015). Mulheres vítimas de violência doméstica: Uma abordagem fenomenológica. Texto \& Contexto-Enfermagem, 24(1), 196-203.

Pereira, A. S., Shitsuka, D. M., Parreira, F. J., \& Shitsuka, R. (2018). Metodologia da pesquisa científica. UFSM.

Reigada, C. L. D. L., \& Smiderle, C. D. A. S. L. (2021). Atenção à saúde da mulher durante a pandemia COVID-19: orientações para o trabalho na APS. Revista Brasileira de Medicina de Família e Comunidade, 16(43), 2535-2535.

Santos, I. B. D., Leite, F. M. C., Amorim, M. H. C., Maciel, P. M. A., \& Gigante, D. P. (2020). Violência contra a mulher na vida: estudo entre usuárias da Atenção Primária. Ciência \& Saúde Coletiva, 25(5), 1935-1946.

Silva, S. D. A., Lucena, K. D. T. D., Deininger, L. D. S. C., H. F. C. C., Vianna, R. P. D. T., \& Anjos, U. U. D. (2015). Análise da violência doméstica na saúde das mulheres. Revista Brasileira de Crescimento e Desenvolvimento Humano, 25(2), 182-186.

Souza, M. B., \& Silva, M. F. S. (2019). Estratégias de enfrentamento de mulheres vítimas de violência doméstica: uma revisão da literatura brasileira. Pensando famílias, 23(1), 153-166.

Vale, S. L. D. L., Medeiros, C. M. R., Cavalcanti, C. D. O., Junqueira, C. C. D. S., \& Souza, L. C. D. (2013). Repercussões psicoemocionais da violência doméstica: Perfil de mulheres na atenção básica. Rev Rene, 14(4), 683-693.

Vasconcelos, M. S., Holanda, V. R., \& Albuquerque, T. T. (2016). Perfil do agressor e fatores associados à violência contra mulheres. Cogitare Enfermagem., $21(1), 1-10$.

Vieira, E. M., Perdona, G. S. C., \& Santos, M. A. (2011). Factors associated with intimate partner physical violence among health service users. Rev Saúde Pública, 45(4), 730-737.

Vigário, C. B., \& Paulino-Pereira, F. C. (2014). Violência contra a mulher: análise da identidade de mulheres que sofrem violência doméstica. Revista de Psicologia, 5(2), 153-172.

Zancan, N., Wassermann, V., \& Lima, G. Q. D. (2013). A violência doméstica a partir do discurso de mulheres agredidas. Pensando Famílias, 17(1), 63-76. 